\title{
Médiévales
}

Langues, Textes, Histoire

47 | automne 2004

Îles du Moyen Âge

\section{Un peuple libre, sauvage et vertueux : nature et politique dans la Corse du Quattrocento}

\section{Antoine Franzini}

\section{(2) OpenEdition}

1 Journals

\section{Édition électronique}

URL : https://journals.openedition.org/medievales/503

DOI : 10.4000/medievales.503

ISSN : $1777-5892$

\section{Éditeur}

Presses universitaires de Vincennes

\section{Édition imprimée}

Date de publication : 1 décembre 2004

Pagination : 63-78

ISBN : 2-84292-157-7

ISSN : 0751-2708

\section{Référence électronique}

Antoine Franzini, « Un peuple libre, sauvage et vertueux : nature et politique dans la Corse du Quattrocento », Médiévales [En ligne], 47 | automne 2004, mis en ligne le 02 novembre 2010, consulté le 24 avril 2022. URL : http://journals.openedition.org/medievales/503 ; DOI : https://doi.org/10.4000/ medievales.503

Ce document a été généré automatiquement le 24 avril 2022.

Tous droits réservés 


\title{
Un peuple libre, sauvage et vertueux : nature et politique dans la Corse du Quattrocento
}

\author{
Antoine Franzini
}

1 Depuis l'Antiquité, les îles semblent avoir été un outil propice et un terrain privilégié pour penser le politique, qu'il s'agisse d'ailleurs de penser l'origine ou le devenir des peuples, sans doute parce qu'elles offrent à l'étude de ces peuples et à celle des territoires qu'ils occupent une figure $d u U^{1}{ }^{1}$. Dans cet esprit, si on s'accorde à remarquer qu'à partir des Grandes Découvertes, les îles seront explicitement l'espace convenu des utopies, on doit constater aussi que, dans les temps précédents, elles ont été implicitement un terrain privilégié pour l'établissement et le développement de l'idée d'une population restée à l'état de nature, un thème dont on sait la fécondité dans l'élaboration de la philosophie politique et du droit moderne ${ }^{2}$. La présente contribution essaye ainsi d'étudier un moment dans ces représentations des îles, grâce à l'exemple de la Corse du Xv ${ }^{\mathrm{e}}$ siècle, à la charnière entre ces deux époques.

2 C'est en effet dès le milieu du $\mathrm{Xv}^{\mathrm{e}}$ siècle qu'on voit se transformer les discours tenus sur la Corse et les Corses, et précisément apparaitre un discours sur la nature des peuples, autrement dit un discours sur le politique, tandis que s'effacent progressivement les discours sur les origines mythiques et l'évocation du merveilleux. Les textes disponibles de Domenico Silvestri ${ }^{3}$ ou Pierre d'Ailly ${ }^{4}$ par exemple, qui illustrent la charnière des $\mathrm{XIV}^{\mathrm{e}}-\mathrm{XV}^{\mathrm{e}}$ siècles et qui faisaient presque exclusivement référence à ces dernières matières (qu'ils reprenaient en général des auteurs anciens à travers Isidore de Séville), font désormais place à de nouveaux points de vue que je vais donc rassembler maintenant ${ }^{5}$.

Dans ces textes, deux opinions dominantes semblent s'opposer à propos de la Corse. Elles reposent l'une et l'autre sur l'idée fondamentale que les peuples qui y vivent n'ont pas encore reçu l'empreinte de la civilisation. Il est d'ailleurs sans doute nécessaire de rappeler, à propos de la nature des peuples, l'influence toujours plus grande sur les lettrés du Quattrocento des références antiques à propos de la Corse, grâce aux 
traductions et éditions d'auteurs grecs dans les années 1450. L'image idéale des Corses forgée par les historiens grecs (Hérodote, Polybe, Diodore de Sicile ${ }^{6}$ y côtoie les préjugés très sévères des auteurs grecs ou latins de l'époque impériale (Strabon, Sénèque, Pomponius Mela ${ }^{7}$ ) à leur endroit. Pourtant, ces deux opinions ne s'opposent pas seulement sur la nature de ces peuples - leur sauvagerie, leur perversité, ou au contraire leur vertu-, bien que ces qualités prennent selon les cas plus ou moins d'importance. Elles s'opposent surtout sur la façon dont il convient de penser leur gouvernement.

4 La première de ces thèses, celle que je vais étudier dans cette contribution, suggère, dans la tradition stoïcienne, que les Corses, soumis à la loi naturelle et restés dans une merveilleuse simplicité, témoignent certes de l'innocence de l'humanité primitive, mais surtout manquent de la sagesse que le philosophe ou le lettré pourrait seul leur apporter. On reconnaitra dans ce dernier trait aussi bien un des fondements des utopies, en particulier celle de Thomas More, que le rôle crucial assigné par Rousseau à la figure du législateur ${ }^{8}$. Je m'attacherai aussi à reconnaître dans ce courant d'idées l'influence discrète, mais insistante, du thème de l'âge d'or, qu'il est parfois difficile de distinguer (si on doit le faire) de ceux de l'abondance ou de la vertu de la vie paysanne. La seconde de ces thèses au contraire, dont je ne ferai pas l'étude ici, et que défendent en particulier les humanistes génois en ce qui concerne la Corse, considère que cet état de nature, en général plutôt jugé pervers ou pour le moins sauvage, mérite seulement de recevoir enfin les lois bienfaisantes de la cité9. L'Église pourrait sans doute civiliser ces peuples et tenir le rôle du maître, mais dans cette île, "l'incroyable et barbare ignorance des clercs", selon l'expression des gouvernants génois, entretient "les abusives, perverses et détestables coutumes corses" (abusos et pravas ac detestabiles consuetudines corsicanas) ${ }^{10}$. En définitive, seules les armes peuvent assurer l'influence bienfaisante de la cité et permettre alors la mise en valeur du territoire. Autrement dit, les uns pensent que la nature de ces peuples est féconde et dispose d'une aptitude naturelle à recevoir l'enseignement du sage, du législateur ou du philosophe. Les autres pensent au contraire que leur nature sauvage ne peut être domptée que par les armes et la contrainte. Certes, dans tous les siècles, les stéréotypes du «bon sauvage » - par exemple déjà présents chez Tacite à la fin du $\mathrm{I}^{\mathrm{er}}$ siècle - alternent avec ceux du barbare destructeur, violent, et surtout différent, dans sa manière de se vêtir, de combattre, dans ses croyances et dans ses lois. Toutefois, une des vertus essentielles de ces diverses mises en représentation semble au Quattrocento d'assurer, de reprendre ou de renouveler le concept politique de peuple (popolo). Dans un contexte régional jusque là largement dominé par les expériences communales et féodales et par l'enseignement chrétien, la question de l'État se pose d'une manière de plus en plus pressante, sous la domination du prince ou des républiques aristocratiques, et avec elle la question du popolo.

5 J'étudierai trois auteurs, écrivant en latin : Poggio Bracciolini, en 1439, et Antonio Ivani, en 1463-1464, pour le genre de la lettre, Pietro Cirneo dans les années 1480-1490, pour celui de l'essai historique. Cet échelonnement temporel devrait nous permettre de saisir l'évolution du thème, et nous garderons à l'esprit, en point de fuite, la construction et les thèmes de l'Utopie de Thomas More, éditée, rappelons-le, en 1516. 


\section{La sauvagerie fécondée par l'humanisme}

6 Commençons donc par la lecture d'une des deux lettres adressées en Corse en mars 1439 par le Florentin Poggio Bracciolini à l'humaniste ligure Bartolomeo Guasco ${ }^{11}$. Âgé de près de soixante ans, Poggio Bracciolini, qui a passé la plus grande partie de sa vie adulte à la curie romaine, avait fondé une famille trois années plus tôt et jouissait d'un loisir tranquille en Toscane ${ }^{12}$. De son côté, Bartolomeo Guasco, d'une quinzaine d'années son cadet, occupait dans l'île la fonction de commissaire de la commune génoise, peu avant l'arrivée du nouveau gouverneur, Giano Fregoso, dont il avait d'ailleurs été le précepteur à Sarzana. En effet, sa carrière était intimement liée à la fortune de la famille génoise Fregoso ${ }^{13}$. Voici la première moitié de cette lettre ${ }^{14}$ :

Alors que je demandais très souvent de tes nouvelles, en raison de l'intérêt que je te porte, auprès de gens dont je me doutais que tu leur étais connu et cher, j'ai appris que depuis longtemps, et même depuis beaucoup plus longtemps que ton humanité ne peut le supporter, tu te trouvais chez les Corses, peuple sauvage et inhumain. Je me demandais avec étonnement, dans la mesure où tu es un homme totalement voué depuis son plus jeune âge à nos études, c'est-à-dire les humanités, ce que signifiait un si long séjour chez des hommes aussi barbares : commander à ces hommes, et à plus forte raison, être à leur tête, voilà ce que j'estimerais être la plus misérable des servitudes. Mais à mon idée, tu évolues parmi ces Corses comme les abeilles parmi les buissons de ronces, où elles récoltent le miel pour en remplir leurs ruches. [...]. Florence, le 18 mars [1439].

Cette lettre, imprégnée du ton facétieux et libre qui est si particulier à Poggio, et avec lequel il interpellait un ami, reprend certes à la fois la réflexion sur le rôle de l'argent et sur l'implication du lettré dans la vie de la cité. «Peuple sauvage et inhumain » (gens fera atque inhumana), les Corses étaient certes d'abord ici le prétexte à une courte dissertation sur les vertus de l'étude et sur l'abjection du gain d'argent, de la cupidité, de la cieca cupidigia de Dante, racine de tout mal. Mais Poggio soutenait aussi que l'humaniste, voué naturellement au retrait et à l'étude, pouvait féconder de son savoir l'administration des "provinces", c'est-à-dire de ces lointains peuplés d'hommes sauvages, restés à l'écart de la culture. Un peuple formé «d'hommes si barbares » que son gouvernement même serait pour Poggio la " plus misérable des servitudes » était-il seulement le prétexte à constituer le contrepoint de la "dévotion à nos études " $^{15}$ ? Il semble dire que le véritable contraire des études, c'est l'argent, "la dévotion à l'argent", et non la sauvagerie, une opposition que soulignait d'ailleurs sans doute l'emploi symétrique du même verbe dedere dans ces deux projets ${ }^{16}$. Sans doute Poggio ne semble pas encore entrevoir une société politique dans ce peuple sauvage. Mais avec la belle métaphore de cette ingrate administration, «[...] à mon idée, tu évolues parmi ces Corses comme les abeilles parmi les buissons de ronces, où elles récoltent le miel pour en remplir leurs ruches ", il semble reprendre un lieu commun éprouvé par les stoïciens, non pas celui d'un peuple païen et innocent qu'il faudrait convertir, ni celui d'un peuple corrompu par le péché qu'il faudrait réformer, mais celui d'un peuple sauvage à féconder par le savoir de l'humaniste.

8 Avec cette référence implicite à Virgile, à l'exaltation des abeilles dans le livre IV des Géorgiques, ne faisait-il pas aussi allusion à sa façon, au mythe de l'âge d'or, à ce moment où la ronce distille le miel, où les animaux vivent en paix, où la nature généreuse et abondante donne ses fruits sans travail, où surtout les hommes cultivent l'amitié, la concorde, la justice, dans une totale communauté ${ }^{17}$ ? S'il se trouve que le miel appartenait depuis longtemps aux représentations obligées de la Corse, son 
amertume et son âpreté le disputaient presque toujours à son abondance. "Abondant " en effet dans l'île pour Diodore de Sicile, comme pour Lukos de Rhégion ou Étienne de Byzance (qui y voyait la cause de la longévité des insulaires), il était aussi «amer et âpre, car sentant le buis » pour Pline l'Ancien (et Théophraste), et par là « infâme » aux yeux d'Ovide. Virgile lui-même, dans la IX Bucolique, faisait dire au pâtre Lycidas, " criaillant », comme il dit, " telle une oie parmi les cygnes harmonieux » ${ }^{18}:$ " Eh bien ! Puissent tes essaims éviter les ifs de Cyrnos [Corse] ! Puissent tes vaches, en paissant le cytise, gonfler leurs mamelles !». La ronce y distillait sans doute beaucoup de miel, par la vertu des abeilles, mais les buis et les ifs de l'île semblaient devoir en gâter la saveur. Si l'abeille était bien pour Poggio, dans ce milieu du Quattrocento, le modèle du lettré, l'humaniste installait dans ce dispositif exemplaire, entre le lettré et le puissant, un troisième terme, ce peuple sauvage et inhumain, à la saveur encore amère. Avec Poggio, l'humaniste prenait sa place dans cette nature, il la fécondait lui-même pour faire advenir, abeille récoltant le miel parmi les buissons de ronce, un nouvel âge d'or ${ }^{19}$.

\section{Un peuple vertueux pour un prince vertueux}

Avec l'humaniste Antonio Ivani, qui écrivait vingt-sept années après Poggio, et qui était son cadet de plus de cinquante ans, les idées développées par Poggio sur la nature du peuple qui habitait la Corse prennent une nouvelle dimension. Homme de confiance des Fregoso (de la branche des seigneurs de Sarzana et de Corse) et précepteur de leurs enfants comme l'avait été Bartolomeo Guasco, il avait reçu en décembre 1463 la charge du vicariat de cette île. Cette charge était réservée à un docteur en droit pour assumer l'exercice de la justice, mais aussi dans ce cas, pour y administrer l'île en l'absence du fils du déjà nommé Giano Fregoso, Tommasino, qui venait de s'en emparer aux dépens de l'Office génois de Saint-Georges. Il est utile de signaler que les quatre lettres que je vais maintenant évoquer sont toutes adressées indirectement au duc de Milan, Francesco Sforza, à travers son secrétaire Cecco Simonetta ou son représentant à Florence, Nicodemo Tranchedino ${ }^{20}$.

Antonio Ivani, alors âgé de trente-trois ans, s'embarquait à contrecœur le 9 décembre sur une birème à destination de la Corse. Dans la lettre qu'il adressait quelques instants avant son départ à Nicodemo Tranchedino, il exprimait combien il se sentait précipité contre sa volonté dans une aventure tout à fait contraire à ses goûts et à ses projets. En voici un large extrait ${ }^{21}$ :

Après vous avoir écrit dans mes dernières et bavardes lettres [...] que je passais en Corse pour une retraite soudaine, je m'embarque presque à l'instant sur une birème. Pour ma part, je ne pensais en aucune manière qu'on me jugerait utile pour maintenir les habitants de l'île dans la loyauté, alors que je suis étranger à leur mode de vie et à leurs usages. Il me sera nécessaire, je le sais, d'entreprendre une chose des plus difficiles: aller contre ma nature et me faire autre que je n'ai été jusque-là. Un coup du sort aussi malheureux et aussi inattendu m'afflige quand j'attendais une vie moins exposée et plus honorable parmi vous. [...]. Pise, le 9 décembre 1463.

11 On retrouve sans nuances dans cette première lettre le lieu commun présent dans la correspondance de Poggio Bracciolini: le lettré, victime des nécessités de la vie mondaine, pouvoir ou argent, est contraint à une mission sans confort parmi un peuple dont il se sent si «étranger à leur mode de vie et à leurs usages». L'humour, sensible dans tout le cours de la lettre, souligne grâce à la chute la nature de cette île: «Vos 
lettres me seront en effet toujours très chères, comme si elles descendaient du paradis jusque dans les limbes".

Trois mois, puis sept mois ont passé lorsqu'il pose sur la société insulaire, dans deux grandes lettres, un regard plus politique, et surtout plus informé. Son discours est sans doute sous-tendu par ses opinions : Antonio Ivani n'était pas du parti de la commune génoise, mais tenait au contraire pour les principautés. Riccardo Fubini a consacré une longue étude au ton original qu'il a emprunté pour affronter le mythe communal qui dominait alors, et pour longtemps encore, malgré la constitution des états princiers. Et en effet, ces courriers, adressés dans un contexte respectueusement amical au secrétaire particulier du duc de Milan, Cecco Simonetta, quelques semaines avant la prise de possession de la Corse par Francesco Sforza, et pour la première, environ un mois avant l'entrée des Milanais dans Gênes, étaient imprégnés de sa conviction du bien-fondé d'une principauté dans l'île. D'ailleurs, après avoir quitté la Corse, dans une quatrième lettre adressée à Nicodemo Tranchedino et rédigée à Sarzana en avril 1464, il adressait au serviteur du duc de Milan un signe dans ce sens ${ }^{22}$ :

J'ai quitté la Corse et tous les Corses, après avoir accompli avec diligence tout ce pour quoi j'avais été envoyé. Je suis rentré pour savoir si messire Tommasino avait décidé de revenir dans l'île ; et je ne me repens pas de l'avoir fait. Car je l'ai trouvé l'âme brisée, et les forces qu'on espérait lui voir, anéanties. Ce que voyant, je pense avoir pris la bonne décision. Il est vraiment chéri des habitants de l'île, et il ne faisait aucun doute qu'il aurait remporté une victoire complète s'il avait hâté son retour dans l'île, en personne avec trois cents fantassins, pour s'emparer des forteresses. Il renoncera, je crois, à cause de la dépense et des peines que cela implique. Et les Corses auront à charge de chercher une autre voie de salut plus honorable [...]. Sarzane, le 24 avril 1464.

Aliam, une autre voie de salut n'est pas pour rien dans cette lettre dont le vrai destinataire est le duc de Milan et qui donne aux Corses leur place d'acteur politique, sensible également dans le rôle pris par l'affection que les Corses pouvaient porter à ce jeune et nouveau prince. Tout en faisant allégeance à Francesco Sforza, il indiquait implicitement qu'il revenait au duc de fournir aux Corses un relais à l'action politique de Tommasino dans le projet de reprendre l'île à l'office de Saint-Georges qui en avait depuis dix ans l'administration.

Deux grandes lettres, adressées à Cecco Simoneta, rendent donc compte ensuite de son séjour dans l'île et sont à ce titre les plus précieuses ${ }^{23}$. Elles s'organisent selon deux lignes de force. La première constate, grâce à l'examen des habitants, dans leur caractère, leur apparence, leur morale, leurs comportements, que le peuple corse est certes étranger à toute civilisation, mais elle fait l'éloge de cet état de nature, en raison de la liberté, de la raison et de la justice qui s'y trouveraient naturellement rassemblées. La seconde brosse au contraire le sombre tableau des puissants qui les dirigent, seigneurs guerriers et clergé ignorant, vestiges d'un monde à réformer, les uns et les autres accusés d'ailleurs d'une certaine dépravation sexuelle, dont le peuple est au contraire innocent dans le discours d'Ivani. Choisissons dans ces deux lettres les passages qui concernent le mieux notre sujet. Voici dans la seconde lettre, où il fait réponse aux questions du chancelier du duc de Milan, le constat de cette incivilité ${ }^{4}$ :

Par sa lettre, il semble que votre grandeur désire que je lui précise [...] en quelle activité les Corses prennent davantage de plaisir. [...] Il est pour moi évident que leurs mœurs répugnent tout autant qu'elles sont étrangères à toutes les manières civiles et à l'éclat de la vertu. [...] Il n'y a plus de cités dans l'île depuis longtemps déjà. Quatre places fortes subsistent: Bonifacio, colonie de Gênes à ce qu'on dit, 
Calvi, Biguglia, et San Fiorenzo. Tout le reste, à part quelques forteresses, sont des lieux sans remparts, comme par exemple des bourgs, des villages et des cabanes éparses ; il n'y subsiste aucune trace d'édifice public ou mémorable.

Puis décrivant la richesse du terroir, il souligne le caractère fruste des habitants ${ }^{25}$ :

Voici, dit-on, les productions de l'île: du froment en abondance si la récolte de l'année a été bonne [...]; ils ont beaucoup de vin sur les côtes et en montagne; de l'orge, des châtaignes, du miel, du lin, de l'huile d'olive, des figues, des cédrats, des poissons de diverses espèces, du petit et du gros bétail en grand nombre. La terre est fertile dans la plaine, qui est restreinte, mais elle manque de meilleurs cultivateurs, car ils sont parfaitement nonchalants pour l'agriculture; et de même, peu soignés, barbus, portant des guêtres (ocreati), ils négligent tous les ornements de la vie des hommes.

L'éloge de l'état de nature, en contrepoint, est présent dans les deux lettres. Dans la première, on appréciera aussi la façon dont l'humaniste interroge sa propre place ${ }^{26}$ :

Cette île, pour ses villages et ses bourgs, ne renferme qu'un tout petit nombre de forteresses et de places fortes; un peuple libre a la libre faculté de s'agiter : leurs remparts sont la mer et les escarpements des montagnes; ils ne font la guerre à personne qu'à eux-mêmes et en sont rarement la victime de la part des étrangers, à moins qu'à cause de la rivalité des factions, ils ne leur en livrent eux-mêmes le prétexte. [...] La pratique des avocats n'est pas méprisable : ils agissent sans pièces écrites, et débattent oralement devant le tribunal, sans références aux lois [romaines], d'après les seuls statuts et les arguments de raison; et ne croyez pas qu'ils manquent d'éloquence ou de l'art de dissimuler : ils deviennent orateurs sans [avoir appris] l'action oratoire, jurisconsultes sans [avoir appris] les lois, dialecticiens sans y avoir été formés. J'expérimente cela chaque jour, et maintenant je sais combien il est difficile d'exercer le droit pour une telle multitude, et de se trouver continuellement au milieu de volontés si opposées. Trente mille hommes et peut-être plus sont soumis à ma magistrature et tous vivent librement, ce qui indique que les Corses ne sont pas aussi pervers que beaucoup le pensent : car si quelque endroit de nos contrées était dépourvu de villes, de forteresses et de places fortes bien peuplées, si les habitants de cet endroit jouissaient d'autant de liberté, je ne sais s'ils mèneraient une vie plus digne d'éloges que ces gens. Pour moi, bien que je réside chez des hommes agités et bruyants, et que ce genre de vie me soit assez pénible, j'ai pourtant estimé que c'était le mérite de la vertu d'être au service de ceux qui ont imposé ce fardeau sur mes faibles épaules.

Puis dans la seconde ${ }^{27}$ :

Quant au peuple, tout en étant très fruste, il obéit du moins assez bien à l'honnêteté; il est généreux dans sa pauvreté et il observe la justice, et ses représentants, pourvu qu'ils soient justes, non seulement il les vénère, mais il les tient pour des dieux.

18 La description des habitants de l'île, ou plutôt, comme il dit, de « la masse du peuple » (multitudo plebis), oppose leur être fruste à l'élévation des qualités morales qu'ils possédent. Agités, bruyants, superstitieux et de peu de foi, peu soignés, barbus et allant pieds nus, ce "peuple libre » (liberum genus) a la libre faculté de s'agiter et ne fait la guerre qu'à lui-même. Il donne l'exemple de nombreuses vertus: honnête, généreux dans sa pauvreté, il pratique une justice fondée sur les coutumes et la raison (solis decretis institutis et racionabilibus argumentis), sans référence à la doctrine ni au droit romain. Cette considération de la nature des peuples en matière de justice est d'ailleurs encore plus sensible dans un courrier adressé au même Nicodemo Tranchedino trois ans plus tard, en janvier 1467, où Ivani évoquait incidemment son expérience insulaire. Plutôt que le savoir du jurisconsulte, écrivait-il alors, il avait été nécessaire d'avoir en soi « des parties appropriées aux mœurs des Corses : l'humanité, la patience d'écouter 
et un jugement naturel, tel qu'il convient à leurs causes et à leurs mœurs ${ }^{28}$ ». On remarquera qu'il mettait non seulement le droit naturel au-dessus du droit positif, mais qu'en précisant qu'il devait convenir "à leurs causes et à leurs mœurs", Antonio Ivani s'avançait jusqu'à considérer un droit naturel propre à chaque peuple. Dès la fin de la seconde lettre principale, en 1464, alors qu'il avait déjà quitté la Corse, il avait tourné son regard avec plus de compassion vers ce peuple auprès duquel il avait vécu pendant quelques mois: "Pour ma part, ayant approché ce peuple, et reconsidérant sa condition, écrivait-il donc peu avant de conclure, je m'afflige de ce qu'ils soient nés pour endurer toutes les scélératesses ».

19 Dans le même esprit, l'humaniste se demandait également si les habitants d'une autre contrée de l'Italie, qui vivraient dans un pays comme la Corse, c'est-à-dire sans même une ville qui soit armée de places fortes et de forteresses, mèneraient une vie plus digne d'éloges que les Corses. En effet, prétendait-il, la rareté des places fortes et des forteresses, et donc du contrôle politique et militaire, entretient chez les peuples libres cette libre faculté de s'agiter et de se soulever contre les rapines des seigneurs. Il semble intéressant de noter que le même thème sera repris par le noble Battista Geraldini, originaire d'Amelia (dans les États du pape), gouverneur dans l'île pour le duc de Milan en 1469. "Si en Lombardie, on gouvernait autant de villages de cette façon, sans la crainte des soldats et nonobstant la civilité et les villes fortifiées, ce serait pire qu'en Corse... », écrivait-il de la Bastia au duc, en reprenant l'idée d'une justice naturelle ${ }^{29}$. Notons que rien ne fait explicitement référence dans les courriers cités au thème de l'âge d'or, sinon peut-être dans le maniement des lieux communs louant la raison et la justice naturelles. Antonio Ivani aurait-il à cet effet puiser à la source de Diodore de Sicile, qui venait d'être traduit du grec par Poggio Bracciolini, sur la commande du pape humaniste sarzanais Nicolas $\mathrm{V}^{30}$ ?

Ils se comportent selon la raison et la justice, écrivait Diodore à propos des Corses,

[...] les rayons de miel appartiennent sans conteste au premier qui les trouve [...], les troupeaux sont distingués par des marques; même sans les faire garder, les propriétaires les conservent sans dommage : dans toutes les circonstances de la vie, ils observent merveilleusement la justice.

\section{Un peuple libre, riche d'un destin propre}

Avec Pietro Cirneo, nous changeons de milieu comme d'époque. Après avoir été recteur de petites paroisses rurales insulaires, Pietro Cirneo, qui était né en Corse en 1447 dans le village de Felce d'Alesani, s'établit en Terre Ferme à partir de 1475 et se consacre aux études, spécialement à partir de 1480, date à laquelle, âgé de trente-trois ans, il devint à Venise pendant douze ans l'élève de Benedetto Brugnolo. Il se préparait ainsi à l'écriture des deux ouvrages qu'on lui connaît, publié l'un et l'autre par Lodovico Antonio Muratori dans les Rerum italicarum scriptores: le Commentarius de bello Ferrariensi, qui traitait de la guerre que se firent Venise et Ferrare entre 1482 et 1484 (tome XXI) et le De rebus corsicis (tome XXIV). Pietro, qui emprunte beaucoup aux auteurs grecs et latins, ainsi qu'aux auteurs du Quattrocento, Biondo Flavio et Platina, décrit avec un enthousiasme patriotique les productions de l'île. Il veut, écrit-il, répondre aux mensonges colportés sur la Corse par Strabon, dont les Res geographicae avaient été imprimés à Rome en 1469 ou $1471^{31}$. Voici d'abord quelques passages du De rebus corsicis dans le registre de l'abondance: on y retrouve, développées, les descriptions d'Ivani, enrichies par ailleurs d'un certain souci naturaliste ${ }^{32}$ : 
L'île est arrosée par des rivières et produit d'excellents pâturages. Le sol est fertile en céréales de toute sorte et donne des fruits de toute nature, surtout depuis Bastia jusqu'à la Solenzara. Ce pays est plat, comme nous l'avons dit, riche, et nourrit des hommes plein de courage; on peut en dire autant de toute la partie centrale, y compris la Balagne, c'est-à-dire les provinces dont la réunion forme la Terre de Commune. Cette partie produit en abondance non seulement du blé et de l'orge, mais encore des châtaignes, du vin, du miel, de l'huile, des chevaux très agiles et des troupeaux de gros et de menu bétail. Pour la production du lin, ce sol est sans rival, surtout à Campoloro [c'est le pays de la mère de Pietro]. Le Cap Corse, la partie la plus rocheuse et la moins étendue, produit seulement des figues, de l'huile et du vin. Le Delà des Monts n'a qu'une fertilité médiocre, mais il est riche en troupeaux, car les habitants donnent surtout leurs soins à l'élevage du bétail.

Il décrivait ensuite les "cours d'eau poissonneux", "la pêche abondante dans les étangs ", et aussi les grands arbres, spécialement les ifs, ainsi que le miel, la cire et les arbres fruitiers de toute sorte. Puis après s'être attardé sur les divers animaux, sauvages et domestiques, il évoquait les produits d'exportation ${ }^{33}$ :

On exporte de la Corse dans les îles voisines et sur le continent des feuilles de myrte séchées au soleil, pour la préparation des cuirs; des herbes et des racines d'herbes employées dans la composition des médicaments et des couleurs; des moutons pour la boucherie, des poissons salés, des peaux, des cuirs, des chevaux et des juments agiles, de la toile, du lin, du drap, des châtaignes séchées à la fumée sur la claie, du corail que l'on extrait avec un filet des profondeurs de la mer, du miel, de la cire, des figues, de l'huile, du sel, des raisins secs, du vin de raisins secs, du vin cuit, de l'orge, du blé, des vins excellents, les uns généreux, les autres doux et légers; on exporte encore de la soie. On s'étonne qu'il faille si peu de travail pour que le sol de la Corse produise des fruits en si grande abondance et d'aussi bonne qualité ; car l'île s'approvisionne largement avec ses propres ressources, si l'on excepte le fer que nous sommes obligés de faire venir de l'île d'Elbe, à peu de frais d'ailleurs. Nulle part les vivres ne se vendent moins cher qu'en Corse.

Dans la description idyllique de ce pays d'abondance, ponctuée de citations de Juvénal, Solin, Priscien, Pline ou Virgile, l'opulence le dispute à la beauté. Dans ce paysage déjà inventé, «les îles voisines, offrant partout des perspectives charmantes, [et] les promontoires de la Ligurie et de la Toscane, que l'on aperçoit de l'île, semblent avoir été disposés à dessein par la nature pour charmer les yeux des Corses » (quodam naturae quasi spectaculo exposita, delectationi sint Corsis $)^{34}$. Certes le merveilleux garde une petite place, avec la description traditionnelle de la pierre merveilleuse nommée catochite, ou des béliers qui, «au lieu d'avoir seulement deux cornes, en ont quatre, six et même jusqu'à neuf ». Pourtant, toute cette description prélude à l'énoncé d'un idéal politique qui annonce à bien des égards les thèmes essentiels de l'Utopie de Thomas More. Les paragraphes consacrés à la nature des Corses, à leurs mœurs et à leur pratique politique brossent en effet un tableau d'une cohérence presque parfaite ${ }^{35}$. Certes, Pietro décrit en premier lieu la bravoure, le goût des armes et de la vengeance. Mais n'est-ce pas qu'ils vivent « dans le mépris de l'or et de l'argent, car ils ambitionnent avant tout la gloire et les éloges", tandis que «les commerçants sont peu considérés»? Puis, remontant en quelque sorte l'ordre des causes, il s'attache à faire l'éloge du corps ${ }^{36}$ :

Les Corses sont un peuple (genus) sain de corps, dur à la fatigue, à la faim, au froid, aux veilles; toujours prêts à verser leur sang, d'une sobriété sévère et inflexible, ils mangent peu, et leur boisson et leurs aliments sont fort communs; leur mise est simple et sans recherche. tempérance, la générosité, l'hospitalité, la nature taciturne, le respect des épouses, 
composent un éloge de la simplicité, illustré si l'on veut par cette sorte de raccourci : "Les Corses, élèves de la pauvreté, hôtes de la vertu, se montrent compatissants envers tout le monde ; c'est en restant fidèles aux principes austères de leur éducation qu'ils conservent à la fois leur pauvreté et la générosité de leur cœur ${ }^{37}$. Et c'est ainsi que logiquement, après avoir encore décrit les pratiques d'entraide, les tâches accomplies par les femmes et les funérailles, il évoque enfin la nature du politique ${ }^{38}$ : «Tous les Corses sont libres (liberi), écrit-il, et vivent sous leurs propres lois ». Puis après être passé aussi vite que possible sur les terres seigneuriales, Pietro Cirneo s'attarde sur la partie de l'île qui vivait sous le régime communal. Il dénonce certes les factions, à la façon d'Ivani, mais il s'avance aussi très loin pour confier à «la Corse » (et non plus seulement aux Corses) un destin politique qui lui appartiendrait en propre ${ }^{39}$ :

Elle est tantôt sans chef, tantôt soumise à l'autorité de quelque personnage influent; ou plutôt, c'est un honneur, une marque de déférence que les habitants accordent à ce personnage, comme c'est la coutume et le devoir du menu peuple d'en accorder aux grands, tout en continuant à vivre sous ses propres lois et en conservant sa liberté. [...] Chaque cité [donc les pievi rurales] élit pour un an ses magistrats qui composent un sénat et font, comme dans les cités libres, respecter la loi par tous les citoyens sans distinction. Si la Corse reconnaissait l'autorité d'un seul homme, ou si elle avait une volonté unique, elle serait, selon moi, invincible.

Enfin, si la générosité de la nature insulaire («On s'étonne qu'il faille si peu de travail pour que le sol de la Corse produise des fruits en si grande abondance et d'aussi bonne qualité ») suggère volontiers la présence du mythe de l'âge d'or dans le De rebus corsicis, la lumineuse évocation de l'absence de minerai de fer en Corse, en contrepoint de l'abondance prodigieuse de toutes les autres richesses naturelles, en est une bien élégante illustration.

On reconnaît chez Pietro, comme chez Ivani, une particularité présente dans l'Utopie de More, la faveur accordée au monde rural, simple et généreux, sur la cité, qui apparaîtra finalement chez More comme un univers perverti par le luxe et le négoce. Il est utile de souligner de ce point de vue que les trois auteurs que j'ai présentés vivaient à l'écart du système politique de la Commune de Gênes qu'ils n'appréciaient guère. Gênes était régie depuis 1339 par le régime du dogat à vie qui permettait d'assurer un certain raidissement des institutions des régimes populaires et d'échapper à l'expérience de la domination d'un seul. Ce nouvel ensemble de règles, comme l'a démontré Rodolfo Savelli, visait à sanctionner l'équilibre instable entre le pouvoir des doges et celui des factions, de façon à éviter l'expérience seigneuriale, bref à opposer, le chef d'une seigneurie, au doge lié par les règles et les statuts de la citét ${ }^{40}$.

Or la famille populaire génoise des Fregoso, si présente dans notre exposé, a assuré le plus souvent la charge dogale durant le $\mathrm{xv}^{\mathrm{e}}$ siècle; il n'est pas douteux qu'elle ait imaginé jouer dans cette cité un rôle comparable à celui des familles qui surent s'imposer dans le rôle du prince dans les villes italiennes. On sait d'ailleurs que la république aristocratique génoise serait fondée en 1528 sur le principe de leur exclusion définitive de la cité. Enfin, la proximité que les Fregoso entretenaient avec les milieux humanistes du Quattrocento a toujours été distinguée de la distance que les marchands ligures maintenaient avec les lettrés.

27 Ainsi, d'un auteur à l'autre se précise la définition de la nature du peuple des Corses, et plus ou moins explicitement, les contours d'un projet politique. Peuple seulement sauvage pour Poggio, mais fécondé par le lettré, les Corses assument un véritable rôle d'acteur politique avec Ivani, dans le choix d'un prince qu'ils chériraient. Avec Pietro 
Cirneo enfin, l'île, munie d'une personnalité propre et de la liberté de choisir, pourrait assumer sa liberté politique. L'esprit de ces textes offre la représentation d'un peuple proche de l'état de nature, ou au moins encore rustique et généreux, et digne de recevoir du lettré les outils propres à épanouir sa liberté. Trouvant sa source dans la tradition stoïcienne, il semble préparer la venue de l'Utopie, et plus loin de l'élan rousseauiste. Le rapport vertueux que devrait tisser le prince avec les hommes qui peuplent le territoire de son État semble ainsi se distinguer progressivement des menées aristocratiques de la future république marchande ligure, toute imprégnée des traditions féodales et communales. Sans doute, les États princiers ne tiendront pas cette promesse, mais le rêve antique qui met en scène la fécondation du peuple par le philosophe ou le lettré, certainement repris dans le monde chrétien, et revisité ici dans la seconde moitié du Quattrocento, ne cessera de poursuivre son chemin.

\section{NOTES}

1. S. Vilatte, L'Insularité dans la pensée grecque, Paris, 1991.

2. On lira en particulier la traduction du De Canaria dans ce même numéro.

3. Dans son De insulis, écrit et modifié entre 1385 et 1406, le Florentin Domenico Silvestri reprend plusieurs citations antiques sur la Corse, en général à travers Isidore de Sévillle. Il en ressort l'image d'une terre riche en pâturages, mais aussi celle de l'occulta nature potentia présente dans la merveilleuse pierre de catochite (Domenico Silvestri, De insulis et earum proprietatibus, éd. C. Pecoraro, Atti della Accademia di scienze, lettere e arti di Palermo, ser. IV, vol. XIV, parte seconda : lettere, 1953-1954, fasc. II, p. 82-83, avec mes remerciements à Nathalie Bouloux qui m'a aimablement communiqué ce document).

4. Pierre d'Ailly (1351-1420), Ymago Mundi, édit. de Cambridge (French books before 1601; 117,5), De Sardinia et Corsica, ca. XLVI.

5. Pour autant, on ne cesse pas, comme Henricus Martellus, de recopier les textes anciens sur ces sujets.

6. «Pour la première fois, aux temps heureux de ce très grand pape [Nicolas V, 1447-1455], les Latins, y compris ceux qui ne connaissaient pas le grec, commencèrent à lire Hérodote [...], Thucydide, Xénophon, Polybe, Diodore de Sicile, Appien, Philon d'Alexandrie [...]. Cuvres qui, en réalité, même si elles avaient déjà été traduites auparavant, étaient disponibles dans des traductions telles qu'elles étaient presque incompréhensibles ", dans Giannozzo Manetti, Vita di Niccolò V [Vita ac gestis Nicolai V summi pontificis (1455)], trad. italienne de A. Modigliani, Rome, 1999, p. 124. La traduction en français de ce passage par I. Trappo, dans M. Miglio, «Culture à la cour des papes (XII $-\mathrm{XV}^{\mathrm{e}}$ siècle) ", dans Cultures Italiennes (XII ${ }^{e}-\mathrm{XV}^{e}$ siècle), I. Heullant-Donat (dir.), Paris, 2000, p. 142. Nicolas V commanda aussi à Guarino de Vérone la traduction de Strabon.

7. Le correspondant et compatriote d'Antonio Ivani (un des auteurs étudiés ci-dessous), Nicodemo Tranchedino, possédait par exemple dans sa riche bibliothèque les œuvres de Strabone cosmografo et Pomponius Mela «que lui avait données Pietro de' Medici » (P. F errari, "Una biblioteca pontremolese del secolo XV», Giornale Storico della Lunigiana, IV, 1912-1913, p. 48-55). On n'imprimait cependant qu'entre 1469 et 1472 Strabon et Diodore de Sicile.

8. Pour l'utopie, comme pour l'âge d'or, je dois beaucoup à l'enseignement donné par Yves Hersant dans les séminaires qu'il a tenus sur ces sujets. "C'est Utopus qui amena une foule 
ignorante et rustique à un sommet de culture et de civilisation qu'aucun peuple ne semble avoir atteint actuellement », ou encore « La tradition veut que tous les plans de la ville aient été tracés dès l'origine par Utopus lui-même " (Thomas More, L'Utopie ou le Traité de la meilleure forme de gouvernement, trad. M. Delcourt, Paris, 1987, p. 138 et 145). Citons encore une fois la fameuse phrase de Rousseau: "Il est encore en Europe un peuple capable de législation : c'est l'île de Corse. La valeur et la constance avec laquelle ce brave peuple a su recouvrer et défendre sa liberté, mériterait bien que quelque homme sage lui apprît à la conserver » (Du Contrat social, l. II, chap. X, Euvres complètes, Paris, 1959-1995, III, p. 956-957).

9. Sur ces dernières questions, en dernier lieu, C. Lévy, «Y a-t-il une place pour l'histoire dans les philosophies hellénistiques?", dans Progrès, réaction, décadence dans le Moyen Âge occidental, Actes du colloque international, Paris, 4-5 octobre 2001, Centre d'Études du Moyen Âge, Sorbonne Nouvelle - Paris III, à paraître. L'auteur montre en particulier combien la leçon stoïcienne était ici différente de celles des sophistes (des hommes violents à l'origine), des épicuriens (une race primitive robuste et malheureuse, proche de l'animalité), ou des sceptiques (la bestialité aux origines).

10. Bibliothèque municipale d'Ajaccio, ms. 175.

11. Publiées à de nombreuses reprises depuis 1844 , les deux lettres adressées par Poggio à Bartolomeo Guasco en Corse ont récemment été éditées par H. Harth dans Poggio Bracciolini, Lettere, II, Epistolarum Familiarium Libri, Florence, 1984, p. 349-350 et 353-354. Une seule concerne notre sujet, dont la traduction doit beaucoup à l'aide de Marie-Clotilde Hubert et Marie-Agnès Ruggiu.

12. D'innombrables études sur Poggio Bracciolini. On lira en particulier E. Walser, Poggius Bracciolinus, Leben und Werke, Leipzig-Berlin, 1914 ; E. Bigi et A. Petrucci, "Bracciolini Giacomo », in Dizionario Biografico degli Italiani, XIII, Rome, 1971, et enfin l'introduction de V. Bruez à Le Pogge, Un vieux doit-il se marier?, Paris, 1998, p. XVII-XLI.

13. Plus de détails dans A. Franzini, Politique et société. La Corse du Quattrocento (1433-1483), thèse de doctorat d'histoire médiévale, Université de Corse, février 2003. Quelques études concernent l'humaniste génois Bartolomeo Guasco, bien moins célèbre que Poggio, en particulier celles de R. Cessi et F.Gabotto. On retiendra que l'humaniste avait rejoint le service des Fregoso. Emprisonné pendant sept mois en 1422-1423 après avoir ourdi entre Bologne et Forlì le complot éventé qui devait redonner le pouvoir sur cette dernière ville à Caterina Ordelaffi et à son mari Bartolomeo Fregoso, le père de Giano (le gouverneur de l'île en 1437), il restait auprès d'eux à Sarzana jusqu'à la fin 1428-début 1429. Banni des États milanais, il revint en faveur auprès du doge Tommaso Fregoso, après que ce dernier eut chassé de Gênes en 1435 les Milanais. On le retrouve donc ensuite à nouveau au service des Fregoso, spécialement en Corse en 1437.

14. Poggio Bracciolini, Lettere..., op. cit., p. 349-350.

15. On sait en effet à travers ses lettres combien son séjour en Angleterre, en 1421, lui sembla un exil dans une contrée "barbare", c'est-à-dire sans «de bons livres, ni aucune trace de l'Antiquité », in V. Bruez, introd. à Le Pogge, Un vieux..., op. cit., p. XXV.

16. " ... nostris studiis, hoc est humanitatis, ab ineunte etate deditissimus », puis « abiectissimi enim et hominis et consilii est, nummis et questui deditum esse ».

17. Sur ce sujet, H. LEVIN, The Myth of Golden Age in the Renaissance, London, 1969. On connaît, par l'édition de deux lettres de l'humaniste Giovanni Toscanella, comment ce dernier, dans les années 1425-1429, avait «lu » à Florence aux jeunes neveux de Tommaso Fregoso, pendant cinq mois, pour l'enseignement de la langue latine et du style, les Bucoliques, les Géorgiques et l'Énéide (R. Sabbadini, «Giovanni Toscanella », Giornale Ligustico di Archeologia, Storia e Belle Arti, 17, 1890, lett. II et III, p.130-134). Bartolomeo Guasco a sans doute été pendant les mêmes années le précepteur des mêmes ou d'autres jeunes Fregoso à Sarzana.

18. Ces références d'après $O$. Jehasse, Corsica Classica. La Corse dans les textes antiques $d u$ VII siècle avant J.-C. au X siècle de notre ère, Ajaccio, 1986, respectivement, d'après Athénée, II, p. 47 a, livre V, 
14 ; Étymologies, éd. Lindsay, 1989-91 ; Histoire des Plantes, V, 8 ; Histoire Naturelle, XVI, 28 et Amours, I, XII, 7-10. Les vers des Bucoliques, IX, 30-31, dans la traduction d'E. de Saint-Denis (Paris, 1983) sont : « Sic tua cyrneas fugiant examina taxos / sic cytiso pastae distendant ubera uaccae ».

19. Il faut remarquer que l'utilisation de la métaphore du miel appartient aussi à la tradition biblique et scolastique, la providence divine y tenant une place semblable à la providence de la nature : «Du miel et du lait sont sous ta langue » (Cantique des cantiques, IV, 11). Pour Albert le Grand (auteur prétendu), Marie contient le miel céleste, c'est-à-dire le Christ, caché en son sein (De Laudibus Beatae Mariae Virginis libri XII, Strasbourg, Martin Flach, 1493, X, 13).

20. Publiées dès 1866 par A. Bertoloni, A. Ivani Sarzanensis vita, Bologne, 1866, p. 16-20, puis à partir de nouvelles sources par C. Errera, «I Corsi e la Corsica alla fine del secolo XV (Da due epistole di Antonio Ivani) ", Archivio Storico Italiano, s. V, VII, 1891, p. 390-400. Notre traduction a été effectuée avec l'aide de Marie-Agnès Ruggiu. Plusieurs études ont été consacrées à Ivani entre $\mathrm{XIX}^{\mathrm{e}}$ et XXe siècles, et dernièrement par R. Fubini, « Antonio Ivani da Sarzana : un teorizzatore del declino delle autonomie comunali ", dans Egemonia fiorentina e autonomie locali nella Toscana nordoccidentale del primo Rinascimento, Pistoia, 1978, puis dans Italia quattrocentesca. Politica e diplomazia nell'età di Lorenzo il Magnifico, Milan, 1994, p. 136-182.

21. C. ERRERA, « I Corsi... », op. cit., p. 392-393.

22. Ibid., p. 393.

23. Ces lettres ont été rédigées le 9 mars 1464 et le $1^{\text {er }}$ juin 1464 et adressées à Cecco Simonetta, le secrétaire du duc de Milan. Des copies étaient envoyées à Nicodemo Tranchedino, représentant du même duc à Florence.

24. Ibid., p. 396.

25. Ibid., p. 397.

26. Ibid., p. 395.

27. Ibid., p. 397.

28. Firenze, Biblioteca Riccardiana, ms. 834, $\mathrm{f}^{\circ} 189 \mathrm{r}$.

29. R. Musso, "Il dominio sforzesco in Corsica (1464-1481)", Nuova Rivista Storica, I, 78, 1994, fasc. 3, p. 578, d'après Archivio di Stato di Milano, Sforzesco, 436.

30. Livre $V, 13$.

31. D'abord publié en 1732 par L. A. Muratori, dans les Rerum Italicarum scriptores (t. XXIV, p. 408, col. 506), puis traduit vers l'italien en 1834 par G. C. Gregorj, qui faisait une riche introduction, Pietro Cirneo était traduit vers le français par L. A. Letteron sous le titre Petri Cyrnaei, clerici Aleriensis, De rebus corsicis, libri quatuor. Chronique corse de Pietro Cirneo traduite en français par M. l'abbé Letteron, Bulletin de la Société des Sciences Historiques et Naturelles de la Corse, 39-42, 1884, désormais abrégé en PC.

32. PC, p. 26.

33. PC, p. 39-40.

34. PC, p. 40. Relevons chez Pietro Cirneo deux autres incidentes sur le paysage (PC, p. 24) : « Les cités et les châteaux [de la pieve de la Marana] construits sur les hauteurs forment un paysage plein de charme et d'agrément» (civitates oppidaque in locis editis aedificata pulchrum ac laetum reddunt aspectum), et un peu plus loin : «[Aleria] a par devant la mer; à droite, à gauche et par derrière, des cités qui, bâties en des lieux élevés, offrent à l'œil le plus beau et le plus charmant des spectacles " (ante habet mare; a dextra, a tergo et a sinistra civitates quae, in locis editis constructae, jucundum quemdam aspectum pulchrumque ac delectationem praebent).
35. PC, p. 50-62.
36. PC, p. 52 .
37. PC, p. 52-54.
38. PC, p. 60.
39. PC, p. 60-62. 
40. R. Savelli, "Capitula, regulae e pratiche del diritto a Genova tra XIV e XV secolo", dans Statuti, città, territori in Italia e Germania tra medioevo ed età moderna, G. Chittolini et D. Willoweit (dir.), Annali dell'Istituto Storico Italo-Germanico, 30, Bologne, 1991, p. 447-502.

\section{RÉSUMÉS}

Dans la seconde moitié du Quattrocento, les discours tenus sur la Corse et les Corses se transforment. Un discours sur la nature des peuples apparait, tandis que s'effacent progressivement les discours sur les origines mythiques et l'évocation du merveilleux. Appuyées l'une et l'autre sur l'idée fondamentale que ces peuples n'ont pas encore reçu l'empreinte de la civilisation, deux opinions dominantes semblent alors s'opposer dans quelques textes humanistes. Certes, elles s'opposent parfois sur la nature de ces peuples - leur sauvagerie, leur perversité, ou au contraire leur vertu. Mais elles s'opposent surtout sur la façon dont il convient de penser leur gouvernement. Le présent article s'attache à la première de ces thèses, qui suggère que les Corses, soumis à la loi naturelle et d'une merveilleuse simplicité, témoignent certes de l'innocence de l'humanité primitive, mais surtout manquent de la sagesse que le philosophe ou le lettré pourrait seul leur apporter.

A Free, Wild and Virtuous People: Nature and Politics in 15th Century Corsica. During the second half of the 15th century, discourses concerning Corsica and the Corsican people underwent significant change. A new discourse about the nature of indigenous peoples was emerging, as those pertaining to the mythic origins and to the evocation of the fantastic began to disappear. Each based upon the fundamental idea that these populations have not yet been marked by civilization, two main opinions seem to oppose one another in certain humanist texts. Without a doubt, they differ at times with respect to the nature of these populations - their savagery, their perversity or, on the contrary, their virtue. But above all, they differ in their manner of conceptualizing their government. The present article takes up the first of these two positions, suggesting that, under a law that is both natural and of a marvellous simplicity, the Corsicans do, indeed, demonstrate the innocence of primitive humanity but, more importantly, lack the wisdom that only a philosopher or a savant could afford them.

\section{INDEX}

Mots-clés : âge d'or, Corse, état de nature, humanisme, utopie

Keywords : Corsica, Golden Age, Humanism, nature state, utopia

\section{AUTEUR}

\section{ANTOINE FRANZINI}

Université de Corse, UFR Lettres - Langues - Sciences Humaines, F-20250 Corte 